\title{
Activating Relaxation-Controlled Diffusion Mechanisms for Tailored Moisture Resistance of Gelatin-based Bioadhesives for Engineered Wood Products
}

\section{K.M. Hess ${ }^{\mathrm{a}}$ and W.V. Srubar III ${ }^{\mathrm{a} \dagger}$}

${ }^{a}$ Department of Civil, Environmental, and Architectural Engineering, University of Colorado Boulder, ECOT 441 UCB 428, Boulder, Colorado 80309-0428 USA, ${ }^{\dagger}$ Corresponding Author, T +1 303492 2621, F +1 303492 7317, E wsrubar@ colorado.edu

\begin{abstract}
The feasibility of tailoring the moisture resistance of bioadhesives by activating relaxation-controlled diffusion mechanisms is demonstrated herein using gelatin, a hydrophilic biopolymer, as a model biobased resin for engineered wood products. The effect of gelatin-to-water concentration and tannin addition on the governing kinetics of water transport in gelatin-based bioadhesives was investigated in this work. Time-dependent flexural mechanical properties of laminated (a) gelatin and (b) gelatintannin wood veneer composites conditioned at both moderate and high humidity were characterized and compared to oriented strand board and plywood. Results indicate that increases in both gelatin $\underline{\text { and tannin content not only decrease rates of water uptake, volumetric swelling, and maximum }}$ $\underline{\text { moisture contents of gelatin-based resins, but also increasingly induce relaxation-controlled moisture }}$ diffusion behavior, which implies short-term moisture resistance and long-term moisture affinity. This behavior could be leveraged to address both in-service (i.e., strength, stiffness) and out-ofservice (i.e., rapid biodegradation) requirements for engineered wood products.
\end{abstract}

Keywords: A. Resins; B. Environmental degradation; C. Transport phenomena modeling; D. Moisture. 


\subsection{Introduction}

\subsection{Adhesives for Engineered Wood Products}

Approximately $60 \%$ of all adhesives manufactured worldwide are used in the fabrication of engineered wood products [1]. The annual global demand for engineered wood, including oriented strand board (OSB) and plywood, has doubled over the past three decades and is projected to double again to 5.2 billion cubic meters by 2050 , due to factors such as rising populations, stricter building codes, and diminishing supplies of old-growth timber [2].

Engineered wood products are fabricated using predominantly synthetic adhesives, such as phenol formaldehyde (PF), urea formaldehyde (UF), or polymer isocyanates (PMDI) - materials that have been identified as allergens and toxic carcinogens [1,3-5]. The negative effects of formaldehyde and the pressure to decrease reliance on petroleum-based resources have recently prompted a resurgence in research on alternative adhesives from natural, biorenewable sources.

Historically, adhesives derived from animal proteins, including gelatin, were used in wood composites until their replacement by low-cost, high-performing adhesives [6,7]. Comprised mainly of the amino acids proline, glycine and 4-hydroxyproline [8], gelatin is a partially degraded form of collagen, a protein found in the skin and bones of animals [9-11]. Commercial gelatin is obtained via thermochemical degradation of collagen's triple-helix structure into random coils, which are stabilized by hydrogen bonds and covalent crosslinking [11-13]. When dissolved in an aqueous (water) solution at elevated temperatures $\left(>50^{\circ} \mathrm{C}\right)$, gelatin exists in a disordered, relaxed coil formation. However, as the solution cools below $30^{\circ} \mathrm{C}$, gelatin becomes more ordered and partially reforms helical structures similar to its parent collagen.

In addition to advantages such as biorenewability, biodegradability, global abundance, and nontoxicity $[10,11,14,15]$, dehydrated gelatin resins exhibit high elastic moduli, tensile strengths, 
and adhesive properties compared to other biopolymers (e.g., starch, acacia gum) and conventional synthetic adhesives $[5,16,17]$. While the majority of gelatin-based materials are currently used in the food, packaging, pharmaceutical, and biomedical industries $[11,18,19]$, recent studies demonstrate that gelatin exhibits sufficient adhesive binding properties for use as a bioadhesive for engineered wood products [5,17].

\subsection{Improving the Moisture Resistance of Gelatin-based Bioadhesives}

As a hydrophilic biopolymer network, gelatin has a high propensity to swell or shrink due to the absorption or desorption of water [20]. While moisture affinity is preferred for out-of-service biodegradation of engineered wood products, moisture resistance is required, especially in the short term, for temporary applications (e.g., concrete formwork, scaffolding, disaster-relief housing). Given that pure gelatin is well known to uptake moisture and lose mechanical integrity immediately upon exposure to high-humidity or wet environments, in-service moisture resistance requirements render pure gelatin a problematic candidate bioadhesive. However, recent research has explored the incorporation of additives, such as polyols, starch, chitosan, tannins, and oligosaccharides, to improve the moisture resistance of gelatin resins [8].

Tannins are polyphenolic compounds derived from vegetables that have recently been investigated as a potential replacement for hazardous phenols in the adhesives industry. In addition to being nontoxic, renewable, and biobased, tannins have a strong affinity for the amino acid proline found in gelatin. Tannin and gelatin are also soluble in water, which eliminates the need for organic solvents during preparation [8]. In addition to the successful implementation of tannin in other formaldehyde-based wood adhesives (i.e., phenol-formaldehyde) [21], tannin has exhibited compatibility with wood substrates and the potential ability to enhance short- and longterm moisture-resistance in gelatin-based bioadhesive networks. 


\subsection{Scope of Work}

In this study, the feasibility of engineering a bioadhesive resin with initially protracted rates of water uptake followed by rapid water absorption was explored by investigating the effect of gelatin concentration and tannin addition on the governing diffusion behaviors of gelatin-based bioadhesives. First, the moisture diffusion behaviors of gelatin (G) and gelatin-tannin (G-T) bioadhesive resins prepared with varying gelatin-to-water (g/w) and tannin-to-gelatin (T/g) concentrations were experimentally determined. The kinetic diffusion behaviors were classified as Fickian or non-Fickian (i.e., relaxation-controlled) and modeled using empirical constants obtained from the diffusion experiments. To demonstrate improvements in initial moisture resistance of engineered wood composites fabricated with G and G-T resins, the time-dependent flexural mechanical properties of eight-ply laminated gelatin-wood veneer (GWV) composites were characterized and compared to two commercial engineered wood products, namely OSB and plywood, after conditioning samples in both moderate and high humidity conditions.

\subsection{Materials and Methods}

\subsection{Materials}

Gelatin was commercially obtained from Knox (Kraft Foods, Inc.) in granular form. Dried tannin powder derived from the Castanea Sativa chestnut tree was supplied by LD Carlson, Company. White oak wood veneer was supplied by Sauers \& Company Veneers. 7/16" OSB and 7/32" plywood were obtained from a local hardware store.

\subsection{Experimental Methods}

\subsubsection{Gelatin Film Preparation}

A $100 \mathrm{~mL}$ beaker of deionized (DI) water was heated to $60^{\circ} \mathrm{C}$ on a Corning PC-420D hotplate. Powdered gelatin, measured in weight percent of gelatin to water $(\mathrm{g} / \mathrm{w})$, was added to the water 
and allowed to dissolve for 15 minutes under continuous agitation by a magnetic stir bar. For tannin $(\mathrm{T})$ addition, powdered $\mathrm{T}$ was measured in weight percent of gelatin (T/g), added to the $60^{\circ} \mathrm{C}$ gelatin-water solution, and allowed to mix for 45 minutes under continuous agitation.

Each mixture was then poured into a 14 x $8 \mathrm{~cm}$ rectangular form for gelation. Upon gelation (approximately 20 to 60 minutes depending on the $\mathrm{g} / \mathrm{w}$ ratio), the material was removed from the form and placed between two pieces of cheesecloth and two grated plates that were secured with ties to prevent warping as the films dehydrated. The films were cured in ambient conditions at a temperature of $21 \pm 2^{\circ} \mathrm{C}$. Four classes of gelatin films with varying g/w ratios $(10 \%, 20 \%, 30 \%$, and $40 \%)$ and three classes of films with $40 \% \mathrm{~g} / \mathrm{w}$ and varying $\mathrm{T} / \mathrm{g}$ ratios $(2.5 \%, 5 \%$, and $10 \%)$ were prepared. The naming convention for the G films is G10, G20, G30, and G40 for $\mathrm{g} / \mathrm{w}$ ratios of $10 \%, 20 \%, 30 \%$ and $40 \%$, respectively. For the G-T films, the convention is G40-T2.5, G40$\mathrm{T} 5$, and $\mathrm{G} 40-\mathrm{T} 10$ for a $\mathrm{g} / \mathrm{w}$ ratio of $40 \%$ with tannin additions of $2.5 \%, 5 \%$, and $10 \%$ by weight percent of gelatin, respectively.

\subsubsection{Moisture Absorption Behavior of Gelatin-based Films}

The moisture absorption behaviors of the four (4) classes of G films and three (3) classes of G-T films were characterized according to a modified ASTM D5229 test method. The G and G-T films were first prepared as described in Section 2.2.1. After curing for 3 days in ambient conditions, the films were placed in an oven at $60^{\circ} \mathrm{C}$ to remove any free water that remained in the gelatin films. The initial (dry) masses of the specimens were obtained using a Mettler Toledo XS105 DualRange scale in 12-hour time increments until mass loss was negligible ( 1\% change). The films were then laser cut into 15 by $15 \mathrm{~mm}$ squares using an Epilog Legend 36EXT laser system. The specimens were massed and dimensioned using calipers before immersion in DI water at room temperature $\left(21 \pm 1^{\circ} \mathrm{C}\right)$. 
Specimen masses were obtained at subsequent time intervals until equilibrium was reached or the specimen degraded. The moisture content, $M C$, was calculated according to the following:

$$
M C=\frac{m_{t}-m_{0}}{m_{0}} \times 100 \%
$$

where $m_{t}$ is the mass of the swollen sample at a given time, and $m_{0}$ is the initial mass of the sample after preconditioning (dehydrating). The dimensions of samples that reached equilibrium were measured to obtain the final change in volume. Samples were tested in triplicate.

\subsubsection{Laminated Gelatin Wood Veneer (GWV) Composite Preparation}

Laminated GWV composites were prepared with G and G-T resins, namely G40 and G40-T10. The resins were prepared as described in Sections 2.2.1. The GWV composites consisted of eight (8) plies of white oak veneer. A layer of resin was applied to the surface of the wood veneer. A second wood veneer was placed on top and pressed to ensure good bonding over the entire surface. This process repeated until an eight-ply composite had been fabricated. The composite was placed between two grated plates that were tightly secured with ties to prevent warping. The composites were allowed to cure in ambient conditions at a temperature of $21 \pm 2^{\circ} \mathrm{C}$.

\subsubsection{Flexural Mechanical Properties of Engineered Wood Products}

After curing, the laminated GWV composites were trimmed using a table saw to dimensions specified by ASTM D790. The flexure specimens are shown in both plan and section view in Figure 1a and 1b, respectively. The 7/16" OSB and 7/32" plywood were also cut to dimensions specified by ASTM D790. The flexural mechanical properties of all four (4) specimen classes were obtained using a three-point bending test conducted with an Instron 5869 Universal Testing Machine with LabVIEW software. Samples were tested in triplicate. 
(a)

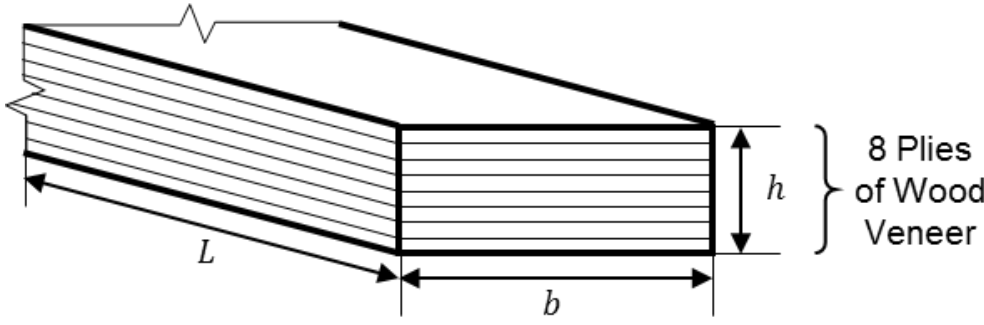

(b)

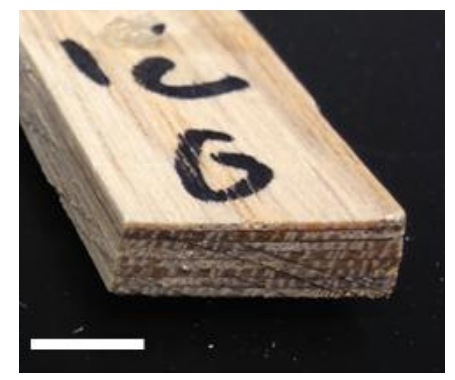

Figure 1: (a) Schematic and (b) cross-section of GWV composites scale bar $=10 \mathrm{~mm}$.

Flexural strength and flexural modulus were determined for each sample. The maximum

flexural strength $(\mathrm{MPa}), \sigma_{f \max }$, was calculated according to the following equation:

$$
\sigma_{f \max }=\frac{3 P_{\max } L}{2 b h^{2}}
$$

where $P_{\max }$ is the maximum applied load $(\mathrm{N}), L$ is the span length $(\mathrm{mm}), b$ is the width (mm) of

calculated according to the following equation:

$$
E_{f}=\frac{C L^{3}}{4 b h^{3}}
$$

where $C$ is the slope of a linear regression based on the load-displacement data between $10 \%$ and $40 \%$ of the maximum applied load and corresponding displacement.

\subsubsection{Time-Dependent Flexural Mechanical Properties in Moderate and High Humidity}

The effects of moderate and high humidity conditioning on the time-dependent flexural mechanical properties of the laminated GWV composites and the engineered wood products (i.e., 7/16" OSB, 7/32" plywood) were investigated herein. Laminated GWV composites were prepared as described in Section 2.2.3 using gelatin (G40) and gelatin-tannin (G40-T10) resins. 
After preparation, the laminated GWV composites were cured in ambient conditions for 7 days. Flexure specimens for all four engineered wood samples were cut to dimensions specified by ASTM D790 using a table saw. The initial flexural mechanical properties of three (3) unconditioned flexure specimens of the laminated GWV composites, OSB, and plywood were characterized according to the methods described in Section 2.2.4. On the same day, twelve (12) flexure specimens of the G40-resin GWV, G40-T10-resin GWV, OSB, and plywood were each placed in both moderate humidity and high humidity chambers. According to ASTM E104, the moderate and high humidity chambers were prepared by placing a supersaturated solution of commercial sodium chloride and sodium phosphate, respectively, in sealed plastic containers that were stored at room temperature. The temperature and humidity in each chamber was recorded using an EL-USB-2-LCD temperature and humidity data logger from Lascar Electronics. For the moderate humidity chamber, the data logger recorded an average relative humidity between $75 \%$ and $81 \%$ and an average temperature of $22.5 \pm 0.74^{\circ} \mathrm{C}$. For the high humidity chamber, the data logger recorded an average relative humidity exceeding $97 \%$ and an average temperature of $22.9 \pm 0.74^{\circ} \mathrm{C}$. Three (3) flexure specimens of all composites were tested after 1, 3, 7, and 14 days of conditioning in the humidity chambers according to the methods described in Section 2.2.4.

\subsection{Theoretical Modeling}

\subsubsection{Classification of Diffusion in Polymer Networks}

The governing moisture diffusion behavior (e.g. Fickian or non-Fickian) of polymeric networks is commonly classified using the power law equation proposed by Alfrey [22]:

$$
\frac{M_{t}}{M_{\infty}}=k t^{n}
$$

where $M_{t}$ is the moisture content at time $t, M_{\infty}$ is the maximum moisture content (MMC), $k$ is a 
kinetic constant characteristic of the polymeric network, and $n$ is a constant that classifies the diffusion mechanism. For a polymer film, $n=0.5$ indicates Fickian diffusion, $0.5>n>1$ indicates anomalous transport, and $n=1$ indicates relaxation-controlled transport.

For Fickian diffusion modeling, the diffusion coefficient, $D$, quantifies the rate at which solvent molecules penetrate a solute. It can be calculated using the following equation:

$$
D_{A}=\frac{\pi}{16} \frac{h^{2} \theta^{2}}{M_{\infty}^{2}}
$$

where $D_{A}$ is the apparent diffusion coefficient, $h$ is the thickness of the specimen (mm), and $\theta$ is

the initial linear slope of the experimental sorption curve $(\% / \sqrt{\mathrm{hr}})$. If the specimen dimensions satisfy a thin-sheet assumption, $D_{A}$ is synonymous with the one-dimensional Fickian diffusion coefficient, $D$; else, an edge correction factor (ECF) must be applied to correctly determine, $D$. For rectangular specimens:

$$
D=\frac{D_{A}}{[1+h / l+h / w]^{2}}
$$

where $l$ and $w$ are the plane length and width dimensions of the specimen, respectively. It should be noted that the ECF, which is defined as the positive square of the quantity in brackets, reduces to unity for a plane sheet with $h \ll<l$ and $h \ll<w$.

With additional assumptions that (1) the material is homogeneous, (2) the moisture diffusion process occurs such that all moisture is transferred through the planar faces and none is transported through the edges, (3) the boundary conditions are constant, and (4) the material has an initially uniform moisture concentration profile, the moisture transport through a plane sheet 
of thickness, $h$, can be expressed as [23]:

$$
\frac{M_{t}}{M_{\infty}}=1-\frac{8}{\pi^{2}} \sum_{n=1}^{\infty} \frac{1}{(2 n-1)} \exp \left[-\frac{D(2 n-1)^{2} \pi^{2} t}{h^{2}}\right]
$$

For anomalous transport, it has been shown that the Fickian diffusion model may not accurately predict the diffusion behavior above $\frac{M_{t}}{M_{\infty}}=0.6$. Instead, diffusion is dominated by relaxation of the polymer network and can be predicted using the following first order BerensHopfenberg differential equation:

$$
\frac{d M_{t}}{d t}=k_{2}\left(M_{\infty}-M_{t}\right)
$$

where $k_{2}$ is the relaxation rate constant. After integration, the solution becomes:

$$
\frac{M_{t}}{M_{\infty}}=1-A e^{-k_{2} t}
$$

where $A$ is a constant [24,25]. Once the constants (i.e., $\left.k, n, k_{2}, A\right)$ are experimentally

determined, the models described by Eq. 4 and Eq. 9 can be combined in a piecewise manner to model anomalous diffusion behavior of water through the polymer network.

\subsection{Results and Discussion}

\subsection{Effect of gelatin concentration on water absorption}

The characteristic water absorption behavior of $\mathrm{G}$ and G-T resins are shown in Figure 2, where the moisture content, $M_{\mathrm{t}}(\%)$, is plotted against square-root-hours $(\sqrt{\mathrm{h}})$. As expected, the resins absorbed water until reaching equilibrium. The data show that the total equilibrium moisture contents of the gelatin samples decreased and that the rates, namely the initial slope $(\theta)$ of the sorption curve, also decreased with increasing g/w ratio. Increasing the gelatin concentration has 
been attributed to an increasingly entangled network [26]. The denser network may prevent the absorption of water resulting in reduced moisture content for higher gelatin concentrations.

As shown in Figure 2, the G10 resin absorbed the most water, reaching an average maximum moisture content (MMC) of 1636\%. One (1) of the G10 samples degraded and could not be measured on the final day. Therefore, the ten-day measurement is based on the average of two (2) samples. The G20 and G30 resins exhibited reduced average MMCs of 1029\% and 1034\%, respectively, while the G40 resin absorbed the least amount of water and reached an average $\underline{\text { MMC }}$ of $1006 \%$.

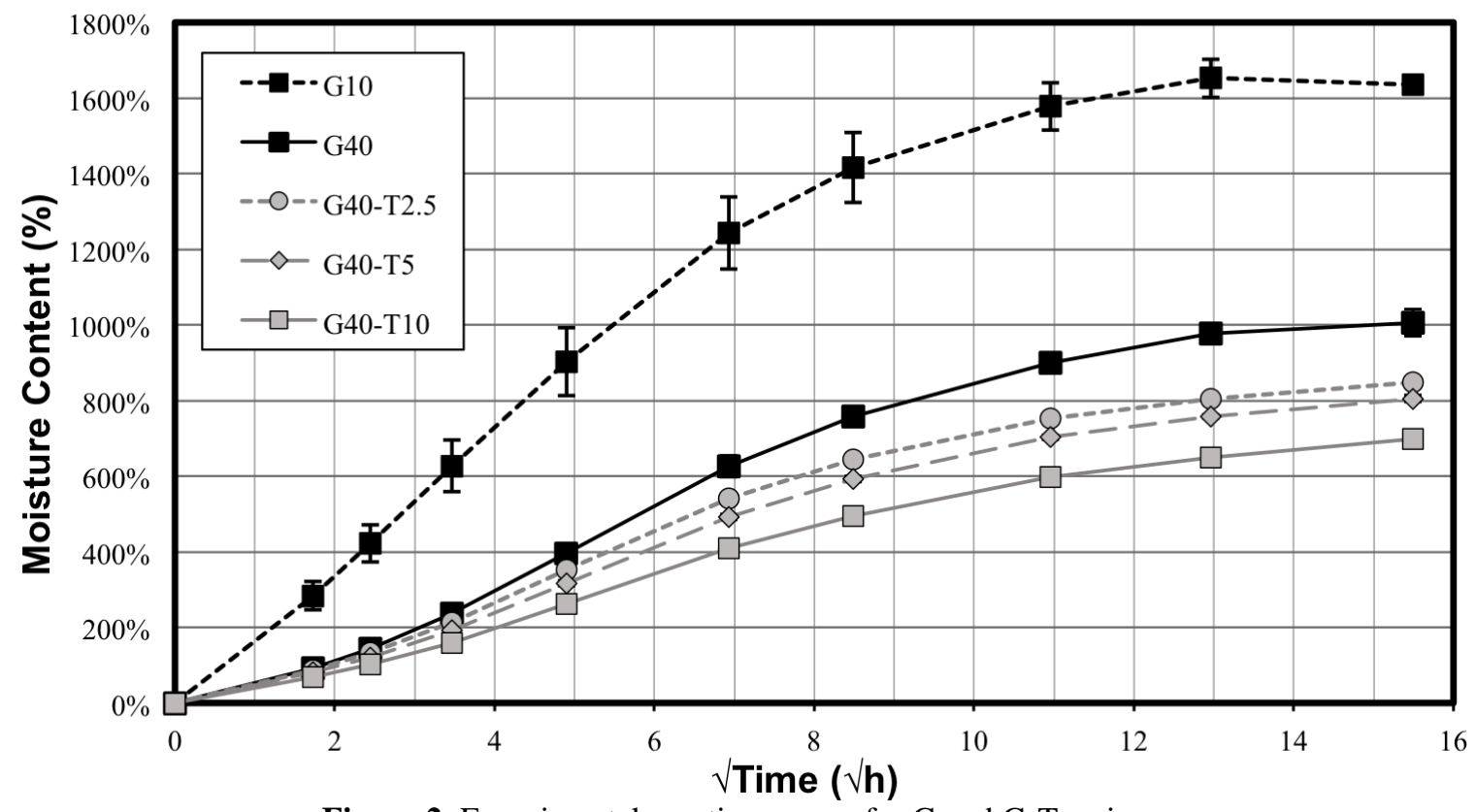

Figure 2. Experimental sorption curves for G and G-T resins.

Although the G20, G30, and G40 resins exhibited comparable MMCs, the G40 sample consistently absorbed less water at each time interval. When compared to the G10 sample, the G40 sample absorbed $38.5 \%$ less water after 10 days. In addition to having the lowest rate $(\theta)$ of moisture uptake, the data suggest that the G40 resin exhibited the greatest overall resistance to water absorption. Thus, G40 was chosen for investigating the influence of tannin addition on the rate and total absorbed moisture of the pure gelatin resins. 


\subsection{Effect of tannin addition on water absorption}

As shown in Figure 2, the G40-T2.5, G40-T5, and G40-T10 resins exhibited decreasing MMCs of $849 \%, 805 \%$, and $698 \%$, respectively, and reduced rates of water uptake with increasing tannin content. When compared to the G40 sample, the G40-T10 sample absorbed 34.8\% and $30.6 \%$ less water after two and ten days, respectively. The tannin addition resulted in a consistent and notable reduction in moisture absorption over time. Pena, et al. [8], suggested that gelatin and tannin are suspected to have two main interactions that improve water resistance. First, hydrogen bonds develop between the tannin hydroxyl groups and the polar groups found in gelatin. Second, hydrophobic interactions may occur to stabilize molecular-level interactions, such as those between proline in gelatin and pentagalloyl glucose in tannin. It is also possible to form covalent cross-links between the gelatin and tannin molecules, which would reduce moisture uptake in gelatin resins.

Table 1 shows the dehydrated (initial) and swollen (final) volumes of the G and G-T samples, along with the total percent increase in volume of each sample. In agreement with the experimental sorption curves, the volumetric responses of the resins suggest that tannin addition reduces total volumetric change of the gelatin-based resins at final equilibrium. The G40-T10 resin exhibited the least amount of volumetric change overall (703\%) and a $44.5 \%$ less volume change when compared to the G40 resin. This improved resistance to moisture may also be attributed to the aforementioned formation of bonds between tannin and gelatin molecules [8].

Table 1. Volume (swelling) change of fully immersed G and G-T resins.

\begin{tabular}{l|c|c|c|c|c}
\hline \multirow{2}{*}{ Sample } & \multicolumn{2}{|c|}{$\begin{array}{c}\text { Initial Volume }\left(\mathbf{V}_{\mathbf{i}}\right) \\
\left(\mathbf{c m}^{\mathbf{3}}\right)\end{array}$} & \multicolumn{2}{|c|}{$\begin{array}{c}\text { Final Volume }\left(\mathbf{V}_{\mathbf{f}}\right) \\
\left(\mathbf{c m}^{\mathbf{3}}\right)\end{array}$} & $\begin{array}{c}\text { Percent } \\
\text { Increase }\end{array}$ \\
\cline { 2 - 5 } & Average & $\begin{array}{c}\text { Standard } \\
\text { Deviation }\end{array}$ & Average & $\begin{array}{c}\text { Standard } \\
\text { Deviation }\end{array}$ & \\
\hline from $\mathbf{V}_{\mathbf{i}}$ to $\left.\mathbf{V}_{\mathbf{f}}\right)$ \\
\hline G40 & 0.80 & 0.061 & 10.91 & 0.297 & $1266 \%$ \\
G40-T2.5 & 0.79 & 0.030 & 8.47 & 0.080 & $973 \%$ \\
G40-T5 & 0.87 & 0.026 & 8.11 & 0.246 & $835 \%$ \\
G40-T10 & 1.03 & 0.013 & 8.30 & 0.184 & $703 \%$ \\
\hline
\end{tabular}




\subsection{Governing Kinetics of Water Absorption}

In order to determine the governing kinetic diffusion behavior of the $\mathrm{G}$ and G-T resins, an analysis of the experimental sorption curves (see Figure 2) was conducted using an empirical fit of the model described by Eq. (4). Rearrangement of Eq. (4) yields:

$$
\log \frac{M_{t}}{M_{\infty}}=\log k+n \log t
$$

A graphical representation of the resulting analysis is provided in Figure 3, which shows the experimental moisture absorption data for $\frac{M_{t}}{M_{\infty}}<0.6$ compared to Eq. (10) by plotting $\log t$ versus $\log \frac{M_{t}}{M_{\infty}}$. Obtained values for the kinetic constants $n$ and $k$ are provided in Table 2 .

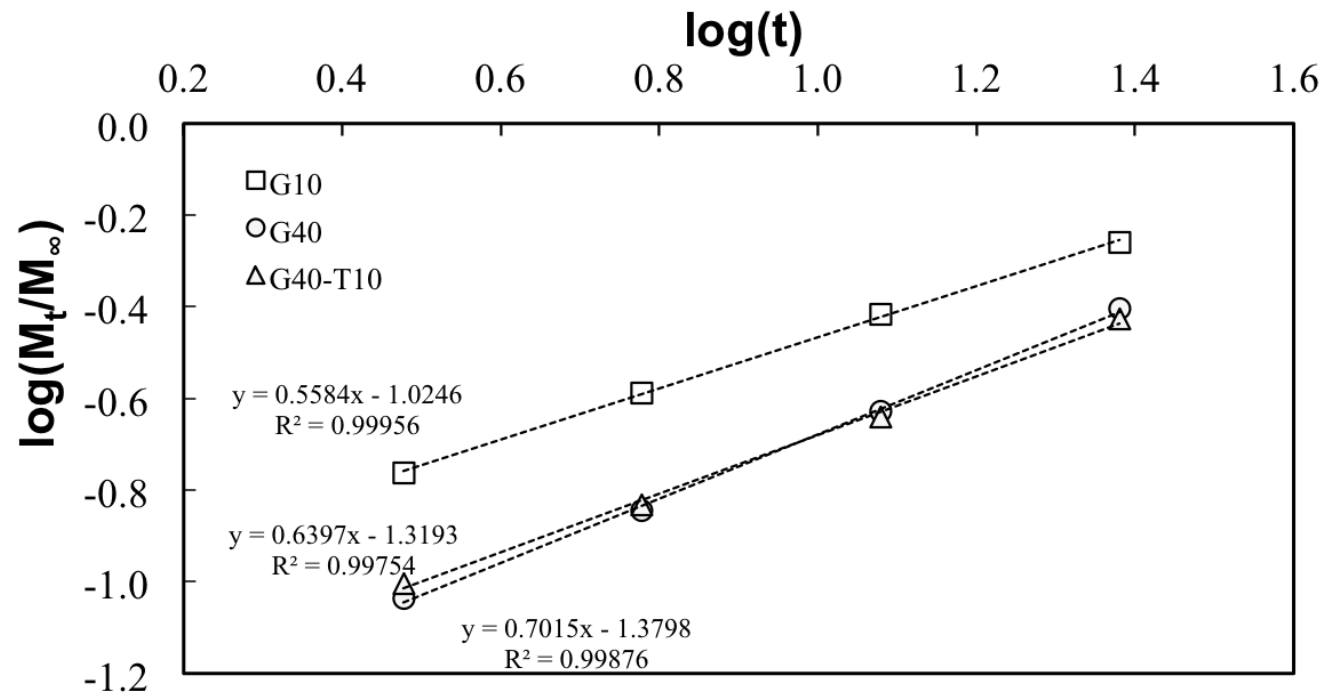

Figure 3. Diffusion case analysis according to Eq. 10 for G and G-T resins.

The G10 resin exhibits behavior closest to the Fickian diffusion case $(n=0.5)$, while, for the G40 and G40-T10 samples, the values of $n$ indicate anomalous diffusion $(0.5<n<1)$. The data in Figure 3 clearly suggest that the diffusion behavior was shifted from Fickian to more 
relaxation-controlled by increases in the $\mathrm{g} / \mathrm{w}$ ratio $(n=0.56 \rightarrow 0.64)$ and further shifted via

tannin addition $(n=0.64 \rightarrow 0.70)$.

Given that that values of $n$ suggest anomalous diffusion for higher $\mathrm{g} / \mathrm{w}$ concentrations and for samples with tannin, further analyses using the Berens-Hopfenberg differential equation, which assumes that the sorption of water depends more on polymer relaxation than penetrant diffusion, was performed to model the diffusion behavior for $\frac{M_{t}}{M_{\infty}}>0.6$. Rearrangement of Eq. (9) yields:

$$
\ln \left(1-\frac{M_{t}}{M_{\infty}}\right)=\ln A-k_{2} t
$$

Figure 4 shows the experimental moisture absorption data for $\frac{M_{t}}{M_{\infty}}>0.6$ compared to Eq. (11) by plotting $t$ versus $\ln \left(1-\frac{M_{t}}{M_{\infty}}\right)$. Obtained values for the kinetic constants $k_{2}$ and $A$ are also provided in Table 2 .

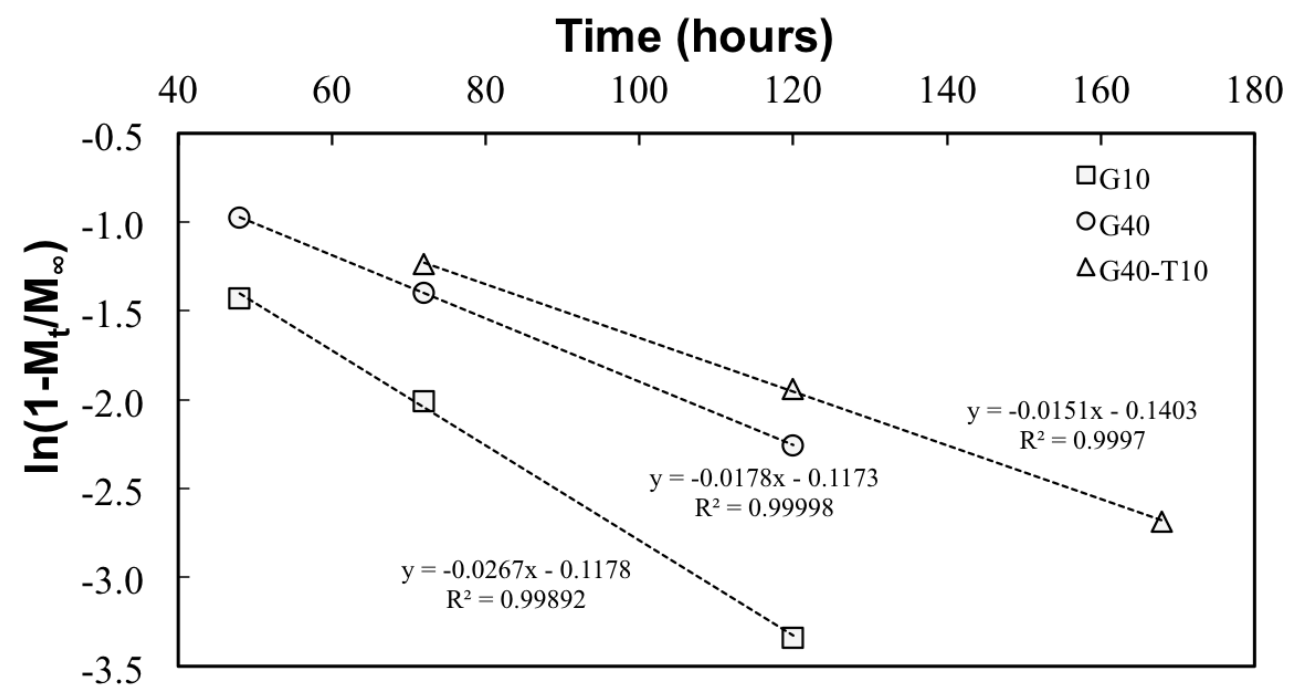

Figure 4. Diffusion case analysis according to Eq. 11 for G and G-T resins. 
The diffusion coefficients and kinetic constants (i.e., $k, n, D, k_{2}, A$ ) for the G10 (Fickian),

G40 (anomalous), and G40-T10 (anomalous) resins were calculated according to the theoretical models presented in Section 2.2.1. The parameters used in the analysis and the diffusion coefficients and kinetic constants are provided in Table 2. For comparison, Wimmer, et al., reported a diffusion coefficient of magnitude $0.00324 \mathrm{~mm}^{2} / \mathrm{hr}$ for fish gelatin adhesives [27].

Table 2. Parameters for diffusion classification and diffusion coefficients and kinetic constants.

\begin{tabular}{l|c|cc|c|c|cr}
\hline Sample & $\mathbf{M}_{\infty}$ & $\mathbf{k}$ & $\mathbf{n}$ & $\begin{array}{c}\text { Diffusion } \\
\text { Classification }\end{array}$ & $\mathbf{D}\left(\mathbf{m m}^{\mathbf{2} / \mathbf{h r})}\right.$ & $\mathbf{k}_{\mathbf{2}}$ & $\mathbf{A}$ \\
\hline G10 & $1636 \%$ & 0.0945 & 0.5584 & Fickian & 0.005 & -- & -- \\
G40 & $1006 \%$ & 0.0417 & 0.7015 & Anomalous & -- & 0.0178 & 0.8893 \\
G40-T10 & $698 \%$ & 0.0479 & 0.6397 & Anomalous & -- & 0.0151 & 0.8691 \\
\hline
\end{tabular}

As shown in Figure 5, the Fickian diffusion model (Eq. 7) can be used to predict the water transport behavior in the G10 samples. In addition, the piecewise anomalous diffusion model can be used to predict the water transport behavior in the G40 and G40-T10 resins. For the piecewise anomalous diffusion model, the Afrey model described by Eq. (4) is used to model the diffusion behavior for $\frac{M_{t}}{M_{\infty}}<0.6$. For $\frac{M_{t}}{M_{\infty}}>0.6$, the Berens-Hopfenberg model in Eq. (9) can be used to describe the diffusion behavior. 


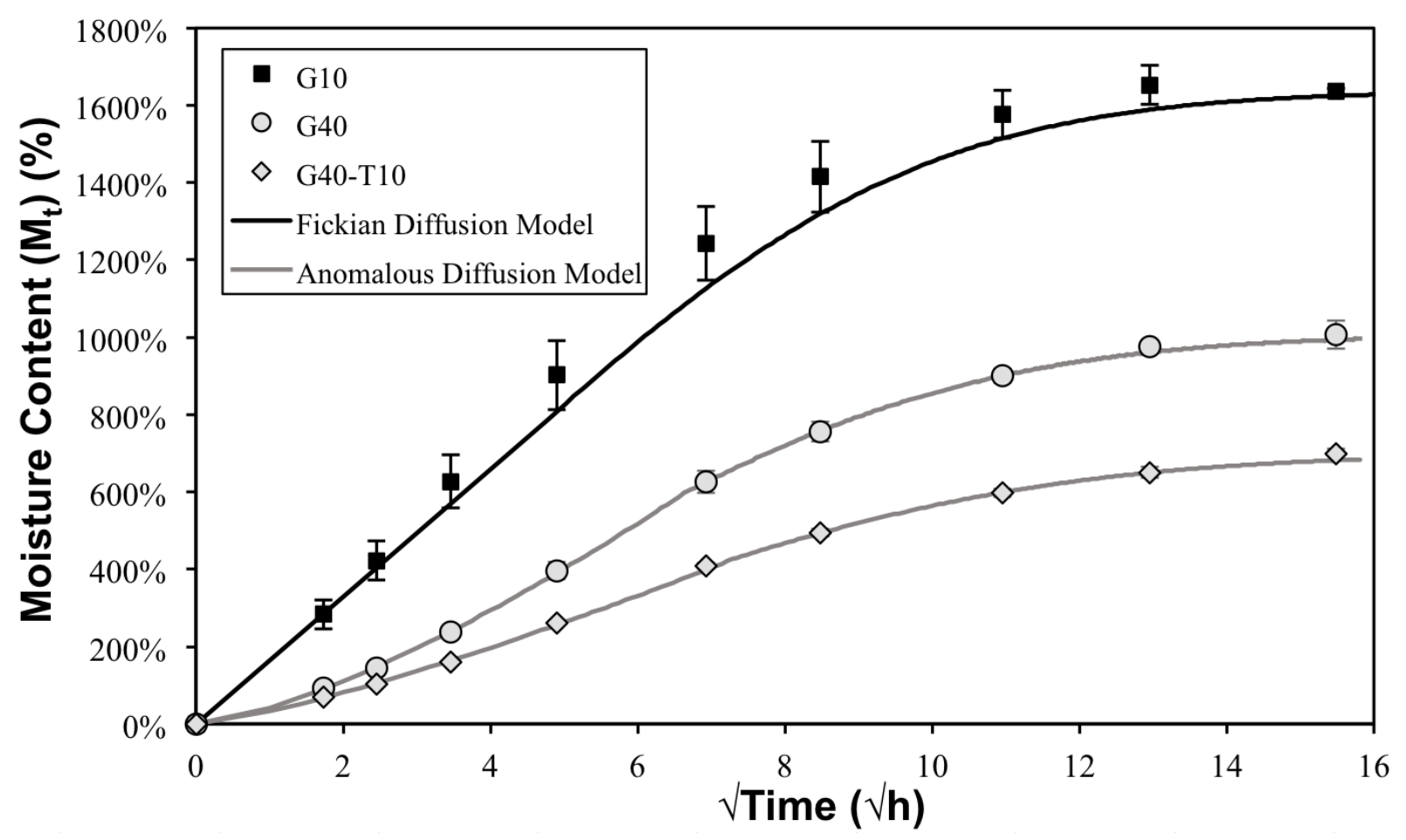

Figure 5. Fickian and anomalous (relaxation-controlled) diffusion model of the experimental sorption curves for G10, G40, and G40-T10 bioadhesive resins.

Figure 5 discerns distinctive behavior between the samples that exhibit Fickian and relaxation-controlled transport. Fickian diffusion exhibits an inflection-free, initially linear rate of water uptake, indicating immediate uptake of water upon immersion, whereas relaxationcontrolled transport exhibits a sigmoidal-shape curve, indicating a brief induction period prior to water uptake. Similarly, Wimmer, et al., measured water sorption and found that the moisture sorption curves of fish gelatin adhesives exhibited sigmoidal-shapes and, as the relative humidity increased, inflection points became more visible [27]. The sigmoidal shape is representative of retarded rates of moisture uptake of the resins at early times (preferred for in-service moisture resistance) and expedited rates at later times (preferred for out-of-service biodegradation).

\subsection{Flexural Properties of Engineered Wood Products in Moderate and High Humidity}

Figures 6 and 7 show the time-dependent flexural mechanical properties, namely flexural strength (modulus of rupture) and modulus of elasticity, for the eight-ply laminated GWV 
composites fabricated with G40 and G40-T10 bioadhesive resins, OSB, and plywood conditioned in moderate and high humidity, respectively.
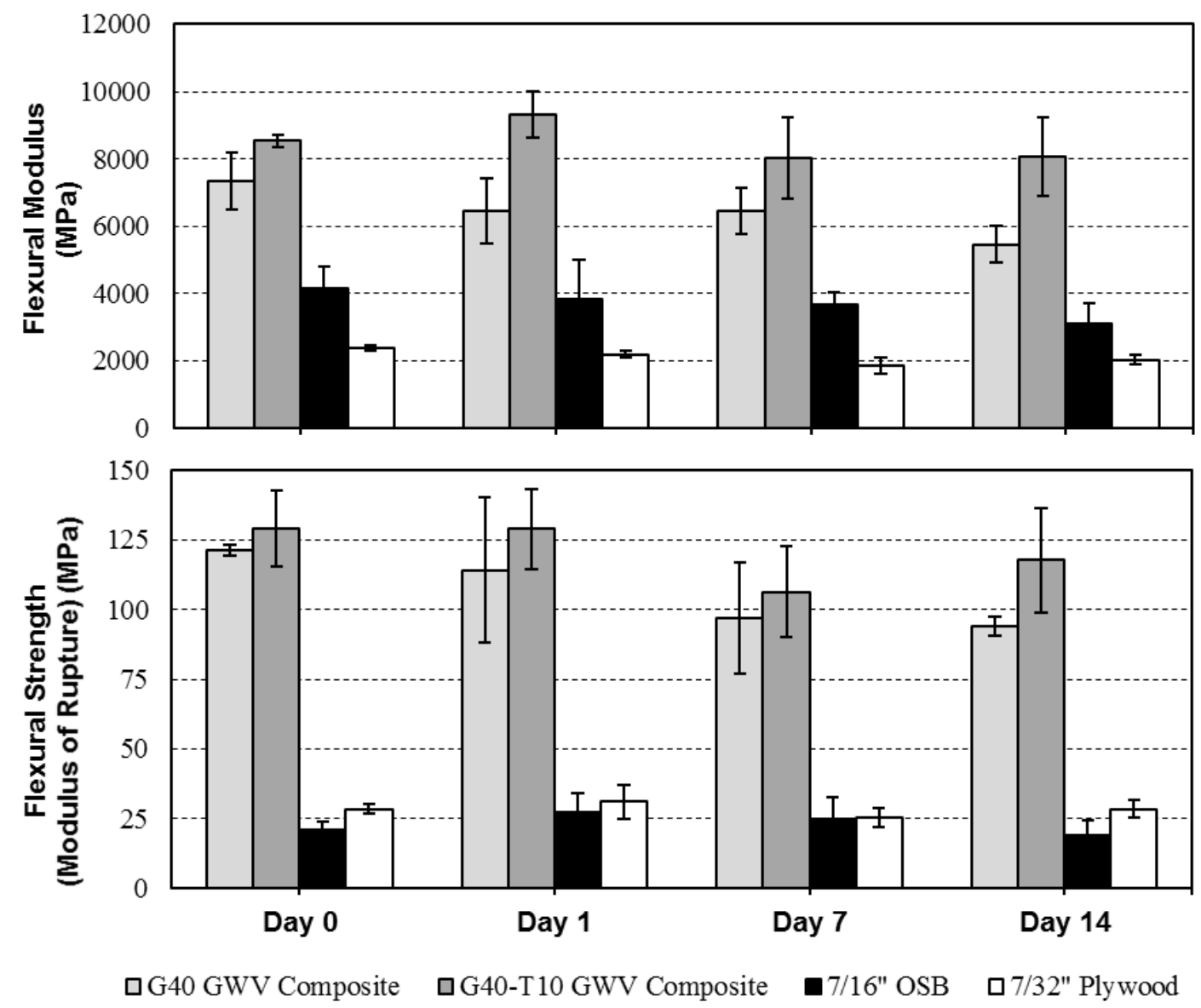

Figure 6. Time-dependent flexural mechanical properties of engineered wood composites conditioned in moderate humidity $(\sim 75-80 \% \mathrm{RH})$.

After 14 days in moderate-humidity conditions, the G40 and G40-T10 GWV composites exhibited total reductions in flexural strength of $22 \%$ and $9 \%$ and reductions in flexural modulus of $26 \%$ and $6 \%$, respectively. In comparison, the OSB exhibited a $9 \%$ reduction in flexural strength, while the plywood exhibited a negligible reduction in flexural strength. Both the OSB and plywood exhibited reductions in flexural modulus of $25 \%$ and $14 \%$, respectively. The laminated GWV composites regularly demonstrated improved initial mechanical properties in comparison to the OSB and plywood. For example, the flexural strength and modulus of the 
G40-T10 samples were 75\% and 394\% greater than the strength and modulus of OSB, respectively, even after fourteen days of conditioning in the moderate-humidity environment. The G40-T10 GWV consistently exhibited improved resistance to property deterioration over time compared to G40 GWV. For instance, after one day of conditioning, the G40-T10 GWV exhibited $6 \%$ and $14 \%$ higher flexural strength and modulus, respectively, compared to the G40 GWV. After fourteen days, this difference increased to $20 \%$ and $32 \%$, respectively.

After fourteen days of conditioning, the flexural strength and flexural modulus of the laminated G40 GWV composites and the laminated G40-T10 composites decreased by $29.0 \%$ and $34.9 \%$ and by $9.6 \%$ and $5.9 \%$, respectively. In contrast, the mechanical properties of OSB and plywood did not significantly decrease as much as the G40 GWV composites, given the high moisture-resistance of conventional phenol-formaldehyde resins. The flexural strength and flexural modulus of OSB and plywood decreased by $10.2 \%$ and $33.6 \%$ and by $0.1 \%$ and $17.3 \%$, respectively. Despite these results, the data demonstrate that the G40-T10 GWV composites $\underline{\text { sustain larger flexural capacities in comparison to the conventional engineered wood products }}$ over 14 days and that these materials could potentially be suitable for temporary applications, provided that the appropriate (moderate humidity) environmental conditions are maintained. 

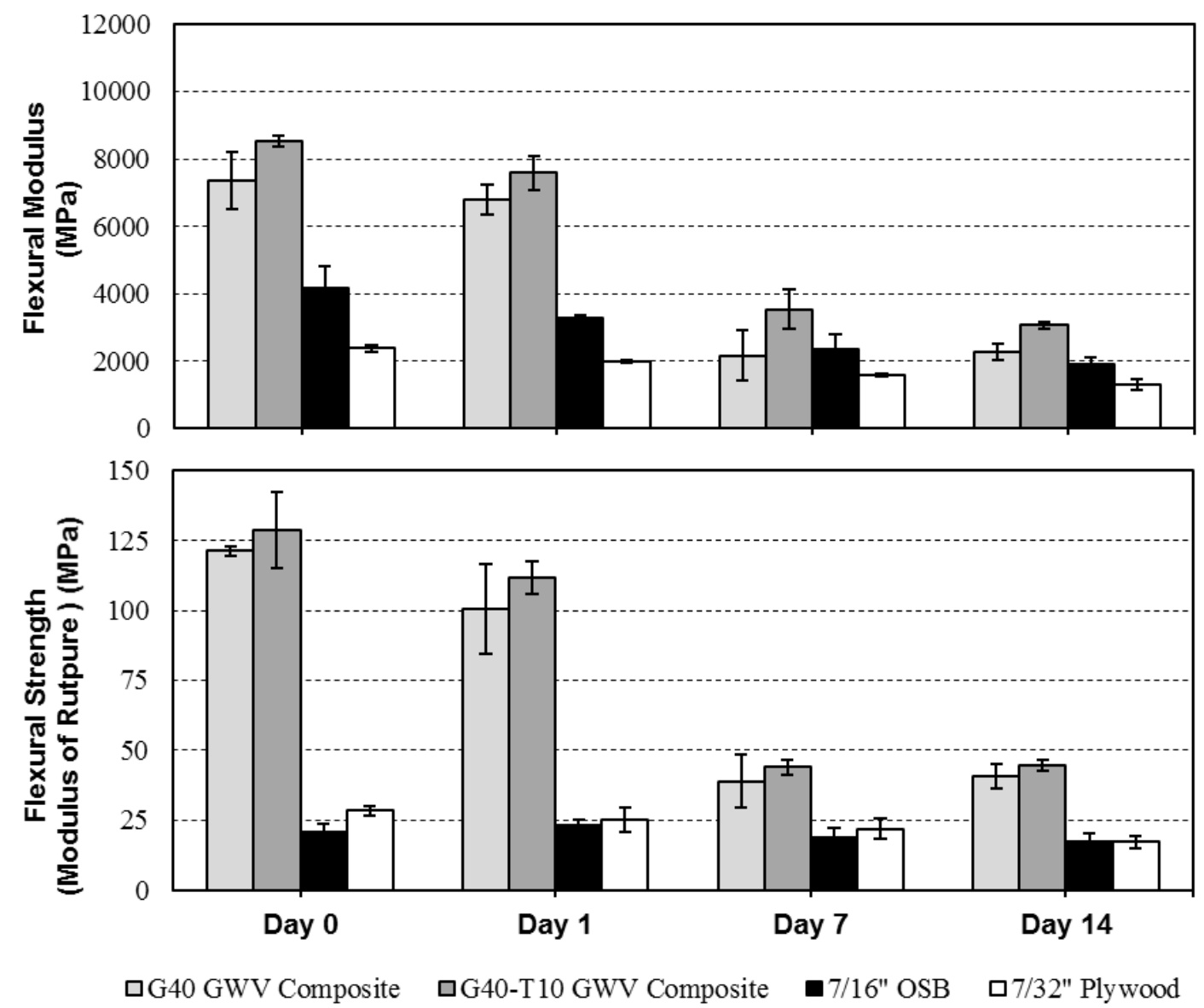

Figure 7. Time-dependent flexural mechanical properties of engineered wood products in high humidity (>97\% $\mathrm{RH})$ conditions.

After one day in high-humidity conditions, the flexural strengths of the G40 and G40-T10 GWV composites decreased $17 \%$ and $13 \%$ and the flexural modulus decreased $8 \%$ and $11 \%$, respectively. In comparison, OSB exhibited an increase in flexural strength by $11 \%$, and the plywood exhibited an $11 \%$ reduction in flexural strength. Both the OSB and plywood exhibited reductions in flexural modulus of $21 \%$ and $16 \%$, respectively. After seven days in high-humidity conditions, G40 and G40-T10 GWV composites exhibited reductions in flexural strength of 66\% and $65 \%$ and reductions in flexural modulus of $69 \%$ and $64 \%$, respectively. In contrast, OSB and the plywood panel only exhibited reductions in flexural strength of $10 \%$ and $23 \%$ and reductions in flexural modulus of $44 \%$ and $33 \%$, respectively. 
These results demonstrate that the laminated GWV composites maintained sufficient flexural properties over a 1-day period but lost structural capacity over a 7-day period in high-humidity conditions. While both OSB and plywood specimens also lost properties after 7 days, these losses were not as significant. As anticipated, the 40G-10T GWV composite performed better than the G40 GWV composite, which can be attributed to the shift from Fickian to anomalous diffusion within the resin and, correspondingly, improved moisture resistance, at least at early times, with tannin addition. Overall, the results show that the GWV composites, especially the G40-T10-resin composite, maintained flexural properties in moderate humidity conditions. While these composites lost flexural properties after one day in high humidity, their residual mechanical properties were comparable to the mechanical properties of conventional engineered wood products (i.e., OSB, plywood) under the same environmental conditions.

For comparison, Triboulet, et al., completed a study that included the performance of gelatin and gelatin-tannin adhesives for wood marquetry applications [28]. The study showed that 2\% tannin addition improved the viscosity (i.e., $>140 \%$ increase for a temperature range of $40-60^{\circ} \mathrm{C}$ ) and tensile strength (i.e., $100 \%$ increase) compared to the pure gelatin adhesive. The tannin additions also increased resistance to moisture and improved the tensile strength (i.e., $>25 \%$ increase) of gelatin-based adhesives after accelerated moisture weathering [28].

Wood-based products are well known to be susceptible to deterioration due to physical and biological decay in the presence of moisture [29]. While this susceptibility to moisture-induced deterioration poses in-service maintenance and structural integrity concerns, rapid deterioration of engineered wood products after disposal (which requires rapid moisture uptake to support biological activity) is a favorable attribute. Rapid post-use degradation minimizes the time needed for biodegradation and reduces recalcitrant volumes of construction material waste. 
Every year, 96 million tons of construction and demolition waste is placed in landfills, and approximately $40 \%$ of this waste is comprised of low-performance materials (e.g., wood, plastics, drywall) [30]. Selecting materials for certain construction applications (e.g., concrete formwork) that are not only designed to last their specific design life but also engineered to degrade rapidly in landfills at the end of their useable life is an environmentally conscious strategy that can promote the sustainable management of construction material waste.

\subsection{Conclusions}

The in-service durability advantages of moisture-resistant adhesives for engineered wood products are, in effect, out-of-service disadvantages due to high moisture resistances and, correspondingly, reduced rates of post-use degradation. To simultaneously address issues with in-service and outof-service requirements for moisture resistance and affinity, respectively, the viability of engineering bioadhesive resins with tailored moisture absorption properties for engineered wood products was investigated herein.

In this study, the kinetic moisture absorption behaviors of gelatin $(\mathrm{G})$ and gelatin-tannin (GT) bioadhesive resins were classified, and the experimental data were used to validate and model a shift from Fickian to anomalous (relaxation-controlled) diffusion behavior with increases in gelatin-to-water $(\mathrm{g} / \mathrm{w})$ and tannin-to-gelatin $(\mathrm{T} / \mathrm{g})$ ratio. The time-dependent flexural properties of laminated wood veneer composites fabricated with G40 and G40-T10 resins were collected over 14 days and compared to the performance of two conventional engineered wood products, namely OSB and plywood, after conditioning in moderate and high humidity environments.

The results of this investigation indicated the following:

1. The experimental sorption curves and volumetric measurements showed that (1) as the $\mathrm{g} / \mathrm{w}$ concentration increased, the rates of water uptake, volumetric swelling, and 
maximum moisture contents (MMCs) of the gelatin bioadhesive resins decreased and (2) the rates of uptake, volumetric swelling, and MMCs were further reduced with T/g ratio.

2. Analysis of the kinetic moisture diffusion behavior demonstrated that the G10 films exhibited Fickian diffusion behavior and that this behavior became increasingly anomalous with increased $\mathrm{g} / \mathrm{w}$ and $\mathrm{T} / \mathrm{g}$ ratios, suggesting that relaxation-controlled mechanisms were being activated. Using experimentally obtained kinetic constants, both Fickian (for G10 resins) and piecewise anomalous diffusion models (for G40 and G40T10 resins) were found to fit experimental sorption data with good agreement.

3. Laminated gelatin wood veneer (GWV) composites initially exhibited improved flexural mechanical properties in comparison to OSB and plywood and maintained these flexural properties in moderate humidity $(75-80 \% \mathrm{RH})$ conditions for 14 days. In high humidity (>95\% RH), the laminated GWV composites maintained $11-17 \%$ of their flexural capacity after 1 day, but lost 59-71\% of their flexural capacity between 2 and 7 days. The laminated GWV composites that were fabricated with G40-T10 resins sustained higher capacities, however, than the GWV composites fabricated with tannin-free (G40) resins, further substantiating improvements in moisture resistance with increased tannin content.

4. Provided an appropriate application, such as concrete formwork, moisture resistance in the short-term and moisture affinity in the long-term may prove beneficial for post-use rapid biodegradation of engineered wood products.

\subsection{Acknowledgments}

This research was made possible by the Department of Civil, Environmental, and Architectural Engineering, the College of Engineering and Applied Sciences, and the Sustainable

Infrastructure Materials Laboratory (SIMLab) at the University of Colorado Boulder with partial 
support from the National Science Foundation (Award \#1537194). This work represents the views of the authors and not necessarily those of the sponsors.

\subsection{References}

[1] Aguilera A. Davin P, Eds. Research Developments in Wood Engineering and Technology. IGI Global, 2013.

[2] Lam F. Modern structural wood products. Prog Struct Eng Mat 2001;3(3);238-245.

[3] Jang Y, Huang J, Li K. A new formaldehyde-free wood adhesive from renewable materials. Int J Adhes Adhes 2011;31(7);754-759.

[4] Cheng HN, Dowd MK, He Z. Investigation of modified cottonseed protein adhesives for wood composites. Ind Crop Prod 2013;46:399-403.

[5] Kim JT, Netravali AN. Performance of protein-based wood bioadhesives and development of small-scale test method for characterizing properties of adhesive-bonded wood specimens. J Adhes Sci Tech 2013;27(18-19):2083-2093.

[6] Frihart CR. Wood adhesives: vital for producing most wood products. Forest Prod J 2011;61(1):4-12.

[7] Pizzi A. Wood Adhesives: Chemistry and Technology Vol. 2. New York: CRC P, 1989.

[8] Peña C, De la Caba K, Eceiza A, Ruseckaite R, Mondragon I. Enhancing water repellence and mechanical properties of gelatin films by tannin addition. Bioresource Technol 2010;101(17):6836-6842.

[9] Khan MA, Islam T, Rahman MA, Islam JM, Khan RA, Gafur MA, Mollah MZI, Alam, AKM. Thermal, mechanical and morphological characterization of jute/gelatin composites. Polym-Plast Technol 2010;49(7):742-747. 
[10] Cao N, Fu Y, He J. Preparation and physical properties of soy protein isolate and gelatin composite films. Food Hydrocolloid 2007;21(7):1153-1162.

[11] Gioffrè M, Torricelli P, Panzavolta S, Rubini K, Bigi A. Role of pH on stability and mechanical properties of gelatin films. J Bioact Compat Pol 2012;27(1):67-77.

[12] Jones RT. Gelatin: manufacture and physico-chemical properties. Pharmaceutical P: London, 2004.

[13] Bigi A, Panzavolta S, Rubini K. Relationship between triple-helix content and mechanical properties of gelatin films. Biomaterials 2004;25(25):5675-5680.

[14] Soradech S, Nunthanid J, Limmatvapirat S, Luangtana-Anan M. An approach for the enhancement of the mechanical properties and film coating efficiency of shellac by the formation of composite films based on shellac and gelatin. J Food Eng 2012;108(1):94102.

[15] Leick S, Degen P, Köhler B, Rehage H. Film formation and surface gelation of gelatin molecules at the water/air interface. Physical Chemistry Chemical Physics 2009;11(14):2468-2474.

[16] Arvanitoyannis IS. Formation and properties of collagen and gelatin films and coatings. In: Protein-based Films and Coatings. CRC Press, 2002. p.275-304.

[17] Dorr DN, Frazier SD, Hess KM, Traeger LS, Srubar WV. Bond Strength of Biodegradable Gelatin-Based Wood Adhesives. Journal of Renewable Materials 2015;3(3):195-204.

[18] Hanani ZN, Roos YH, Kerry JP. Use and application of gelatin as potential biodegradable packaging materials for food products. Int J Biol Macromol 2014;71:94-102. 
[19] Duconseille A, Astruc T, Quintana N, Meersman F, Sante-Lhoutellier V. Gelatin structure and composition linked to hard capsule dissolution: A review. Food Hydrocolloid 2015;43:360-376.

[20] Doi M. Soft Matter Physics. Oxford UP, 2013.

[21] Pizzi A, Mittal KL, Eds. Wood adhesives. CRC P, 2011.

[22] Alfrey T, Gurnee EF, Lloyd WG. Diffusion in glassy polymers. J Polym Sci Pol Sym 1966;12(1):249-261).

[23] Srubar III WV, Frank CW, Billington SL. Modeling the kinetics of water transport and hydroexpansion in a lignocellulose-reinforced bacterial copolyester. Polymer 2012;53(11): 2152-2161.

[24] Ganji F, Vasheghani-Farahani S, Vasheghani-Farahani E. Theoretical description of hydrogel swelling: a review. Iran Polym J 2010;19(5), 375-398.

[25] Kim B, La Flamme K, Peppas NA. Dynamic swelling behavior of pH-sensitive anionic hydrogels used for protein delivery. J Appl Polym Sci 2003;89(6):1606-1613.

[26] Djabourov M, Nishinari K, Ross-Murphy SB. Physical gels from biological and synthetic polymers. Cambridge UP, 2013.

[27] Wimmer, R., Kläusler, O., \& Niemz, P. Water sorption mechanisms of commercial wood adhesive films. Wood science and technology 2013;47(4):763-775.

[28] Triboulet, M. C., Lavigne, E., Monteau, L., Boucher, N., Pizzi, A., \& Tekely, P. The restoration of old wood furniture marquetry. Holzforschung und Holzverwertung $\underline{1996 ; 48: 61-65 .}$

[29] Netravali AN, Pastore CM, Eds. Sustainable Composites: Fibers, Resins and Applications. DEStech Publications, Inc., 2014. 
Manuscript Submitted to:

Composites Part A: Applied Science and Manufacturing

[30] US EPA Recover Your Resources: Reduce, Reuse, and Recycle Construction and Demolition Materials at Land Revitalization Projects. EPA-560-F-09-523, 2009. 